International Journal of Engineering \& Technology, $7(2.24)(2018)$ 16-19
International Journal of Engineering \& Technology
WPC
Website www.sciencepubco.com/index.php/IJET
Research paper

\title{
IOT Based Wheeled Robotic Arm
}

\author{
Abhilash V ${ }^{*}$, P.K.Mani² \\ ${ }^{1} P G$ Student, ${ }^{2}$ Associate Professor, \\ ${ }^{1,2}$ Dept. of Electrical and Electronics Engineering, \\ Veltech Multitech Dr. Rangarajan Dr. Sakunthala Engineering College, Avadi, Chennai-25,India \\ * Corresponding author Email: v.abhilash07@gmail.com
}

\begin{abstract}
The Internet of Things (IoT) is one major field which is fast developing now a days. The IoT means the connection or controlling of an equipment using internet. The another field everyone attracted is towards the robotics. Everyone wants to use a robot because they can do things what we can only imagine to do so. Here in this project we are blending the IoT with the robotics. The robot is controlled by the IoT here. The robot used here is the wheeled robot. Because it is easy to use a wheeled robot in a smooth and hard surface, and it is more energy efficient than the legged robot. The basic motive of the robot here is to pic and place an object from one place to another. This paper discuss about the pick and place wheeled robotic arm which is controlling using the Internet of Things.
\end{abstract}

Keywords: RISC, Microcontroller, IOT, WMRs, SRAM, Dual H-bridge Motor Driver integrated circuit.

\section{Introduction}

This Paper explains to create a robotic arm which works as the things humans cannot do repeatedly. The robotics is the study which consists of electrical engineering and mechanical engineering. The robotic arm used here is to pick and place an object. It can be used as a whole robot or it can also use as a part of the big robot. The arm of robot moves forward and backward directions. Also another joint moves left and right directions of the robotic arm. Another one joint connected will be moving as a rotational motion.

The robot used here is the wheeled robot. The wheeled robotic arm is very energy efficient, the wheels can move very freely and quickly through the smooth and hard surfaces. For the legged robots it took time and need more commands to get forward, backward or any direction movements. But in the case of wheeled robot the commands are not complicated and the movements of robot will be very quick. This wheeled robots can be very useful in the industrial purposes. Because in the industries they use smooth and hard surfaces. For the wheels we use motors for the rotation.

The command to the robotic arm is given through the Internet of Things. We can use a mobile phone to transmit the data or give the command to the robot. In the mobile phone we can use an application to control the robotic arm. The application used here is the Blynk. For that we need internet connection to the mobile phone and the robotic arm. In the robotic arm we use Wi-Fi connection for the internet connectivity. The Wi-Fi can be connected to the Arduino microcontroller which we use in the robotic arm. With the Internet of Things we can control the wheels of the robot as well as the arm of the robot. For that we have to upload program commands to the chip of the Arduino microcontroller of the robotic arm. There will be a battery for the connection of power supply to the robotic arm. For the movement of the robotic arm we need some electrical energy that is given by the power supply.

\section{Methodology}

\section{A. Existing Methodology}

In the existing system we have the robotic arm with 4 degree of rotation. Also the control of the Robotic arm is by the remote control or infrared signal. The Robotic Arm we have now can pick and place the Objects but it is restricted due to the lack of access to all surrounding place to it. Because there is only 4 degree of rotation and it can't drive from one place to another to pick or place an object too far from the robotic Arm. There is also a problem of losing security and privacy due to no password or hiding techniques to the control of the Robotic Arm.

\section{B. Proposed Methodology}

In the Proposed methodology the main part of the paper is the robotic arm, which can pick and place things from one place to another. To control this action we can move the robotic arm by giving specific commands. The robotic arm is equipped with servo motors. These motors help to move the arm in desired direction. The motors are controlled with the help of a microcontroller (ARDUINO). The user interface which is used to control the robotic arm is made on an app. The control is given via the internet to the wifi module. This acts as the receiver and gives the received signal to the microcontroller. The signal which is given to the robotic arm is actually sent through the internet and hence we can access the robot from any place. However the app must require a login ID and a password for security reasons, for a particular person to control the robotic arm. The movements which are made by the robotic arm can be recorded and saved. In this way the arm can do the same movements repeatedly whenever needed. 


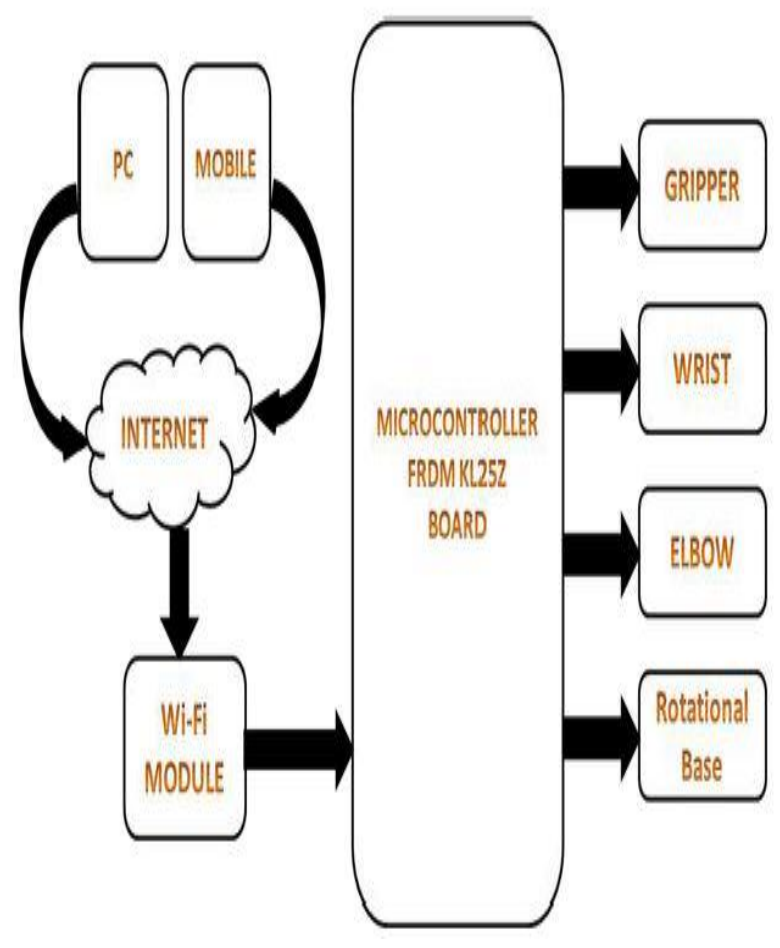

Fig. 1: Block diagram of proposed system

The Proteus 8 Professional software is used for the testing of the working of Iot controlled wheeled robotic arm. This software is created with the modular entities known as the Knowledge Components.

\section{Components Used}

\section{A. Arduino}

The Arduino microcontroller is used to control the whole robotic arm. Here the Arduino controller we use is ATmega328 microcontroller. In the Arduino microcontroller there is a chip where we need to upload all the programs to control the robotic arm. Also there will be a port to connect it with the computer by that port we can upload all the programs we needed. Then there is a port which is used to connect to the internet it can be either connect by the wifi module or by an internet connected computer. The Arduino microcontroller is directly connected to the arms and the wheels of the robotic arm. There is three wires present in the Arduino microcontroller, each have different colors. The colors define the purpose of the wire, one is use to give the input output another is used to connect to the power supply, and the third is used to connect to the controller of the robotic arm. The program used in the Arduino microcontroller is very simple thus we can write it very easily.

\section{B. Motor Driver}

Motor Drivers are the IC used to control the Motors used in a component. The Motor Driver used here is the L293D. The motor driver is used to control the rotation motion of the DC motor. It is fixed to the DC motor in the robotic arm. We use three DC motors in the robot. For each DC motors we use separate motor drivers.

\section{Servo Motors}

There is three wires present in the servo motors which are used to power supply and to the control of the signals to the servo motors. The servo motor is the motor used to make the rotation motion which lead to the movement of the robot's arm. We use more servo motors in the robot's arm than the DC motor because the accuracy of the servo motor is very high than compare to the DC motor.

\section{Gear Motor}

Here we use three DC gear motors. A gear motor is a motor designed with an integral and gear reducer parts. The end shield of the drive end of motor is designed to give dual functions. The side which faces the motor gives the rotor bearing and a sealing through where the integral rotor shaft pin passes. On the other side of the end shield, it gives multiple bearing supports for gearing itself, and also a sealing and fastening provision for the gear housing. This is the process happening in the DC gear motor.

\section{E. Power Supply}

Power supply has very important role in electronic system thus its design will be having a major part in each of the applications. Proper choice of power supply is needed to avoid the maloperation which results to discontinues power supply and fluctuated load. The power supply circuit operates by using the built filters, voltage regulators, and the rectifiers.

The power supply used here is the DC supply which we give individual supply to each of the components in the robotic arm

\section{Software Used}

\section{A. Arduino Software (IDE)}

The Arduino is a hardware microcontroller which we use here is the ATmega328 which is also known as datasheet. It consists of a 14 pin digital input/outputs. Also it has a $16 \mathrm{MHz}$ ceramic resonator, a port for the USB connection, an ICSP header, a power jack and a reset button. The Arduino kit will work when it is connected to a computer with an USB cable or with an AC-to-DC adapter or a battery. Arduino Software or Arduino Integrated Development Environment (IDE) consists of a text editor for writing codes and messages. A text contains a series of menus and toolbar with the buttons for the common functions. It will be then connected to the Arduino hardware to upload the programs and communicate with them. Sketches are the programs written using the Arduino Software or IDE. The sketches or the programs are written in the text editor section. The file extension we use here is .ino. The editor can cut and paste the program. Also can search and replace the text. The feedback will be given in the message area when saving the sketch and exporting it. Also it displays the errors in the sketch if there is. The text output is displayed in the console area of the Arduino Software or IDE. The console message includes the complete error messages and the other information. We can correct the errors and then execute the sketch to run the desired program.

\section{B. BLYNK}

The BLYNK application is a mobile phone application which is used to connect the Arduino with the mobile phone. Using the BLYNK application we can command to the robotic arm to make the robot to work. The things we needed to control the robotic arm by BLYNK is by the internet connection to both the mobile and the Robotic arm. In this application we can arrange the control panel and access and control the controls as we wish. The controls we used in the robotic arm is given below in the figure. 2 . 


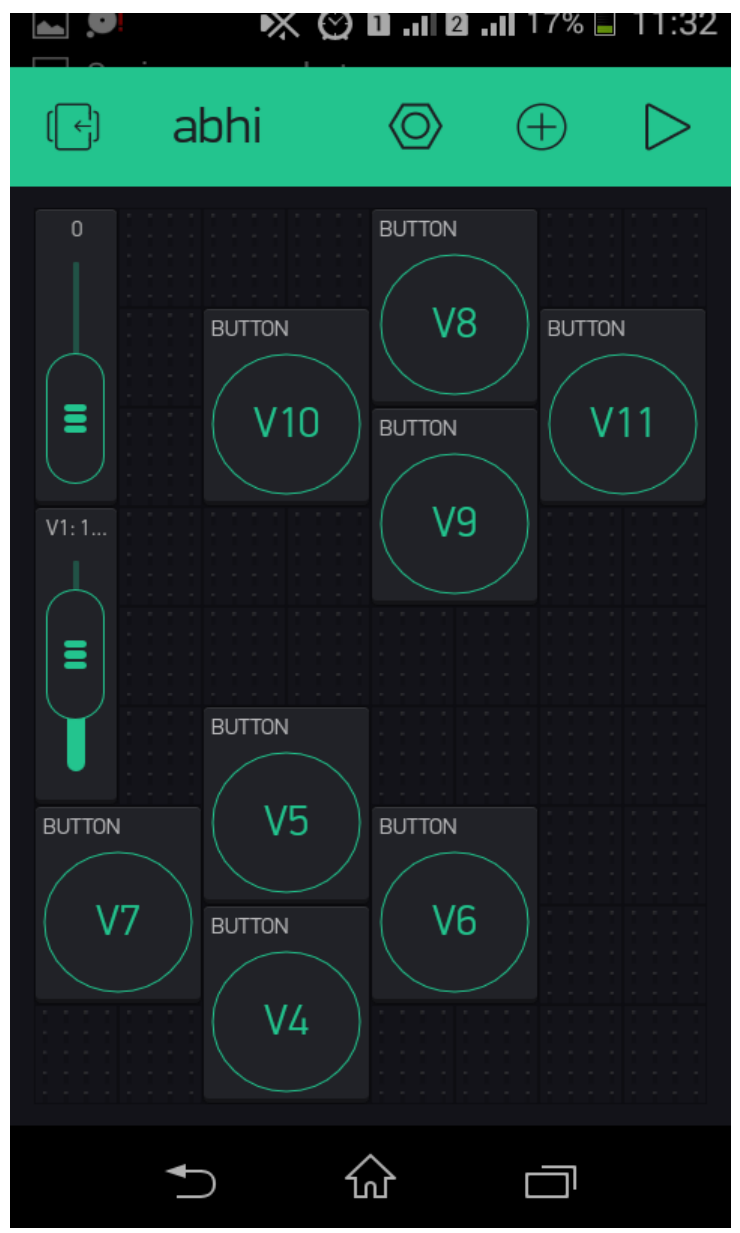

Fig. 2: Blynk Application in Smartphone.

\section{Results and Discussion}

In the earlier studies mainly focus on ROBOT control model by IOT online communication through mobile phone. The robot has lack capabilities to respond in emergencies and Losing security and privacy. Therefore the present work aims to overcome these demerits. Here we discuss about the robotic arm's working, which is controlled by IoT. The complete block diagrams of proposed work is given in figures 3 and 4 .

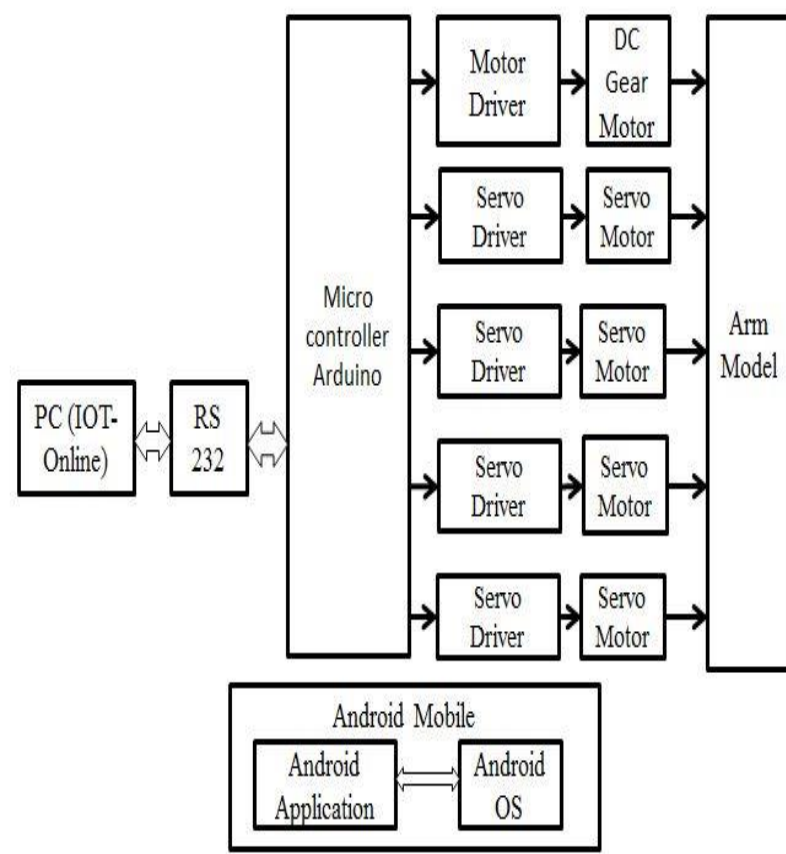

Fig. 3: Block diagram of Robotic Arm

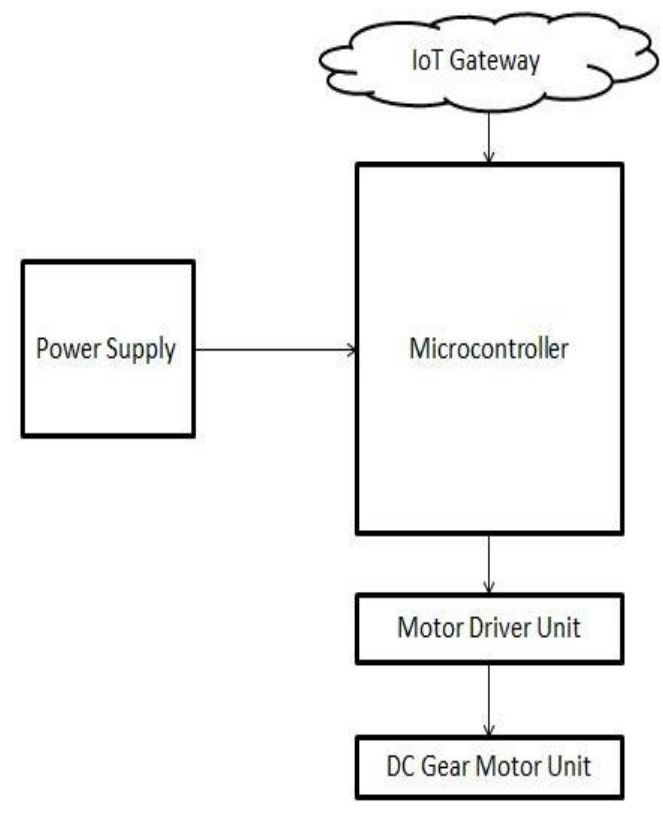

Fig. 4: Block diagram of Robot's wheel.

It is a combination of not only a particular field but a fusion of many fields such as mechanical, electrical, electronic engineering, computer science, technology, math and science. Robots are indispensable in many manufacturing industries. More than this, if it is programmed for a task then the robot performs that task repeatedly with a high accuracy that surpasses even the most experienced human operator. To move like a human arm we need actuator to perform it like a human arm. Actuators are devices which converts the electrical energy into physical motion.

In this robotic arm we are going to use servo motors as servo motors gives good accuracy than any other motors. To look like a human arm we are going to connect this using servo clamps. The servo clamps are of different types, by the help of this various clamps a robotic arm is built. To pick and to put it from one place to another we are going to use a gripper. This gripper is also known as an end effector. There are several types of robot arms are going to use 6 Degree of Freedom it can be less or more as per the requirements.

In order to communicate with the Robotic arm over the internet, we are using Wi-Fi module ESP 8266. It is a self-contained SOC and can be used with any microcontroller to access Wi-Fi network. It consists of 32 bit low powered CPU, and follows 802.11 Wi-Fi standards. It has a powerful storage capabilities and the on board processing, and also it is extremely cost effective.

The ESP module can be directly connected to our KL25Z board. Here we are using serial communication between ESP 8266 and KL25Z. A Wi-Fi based web server is made with a graphical user interface, and controls are given through this GUI. The signals when sent to the module can be given serially to the controller board and then to the servo motors of the robotic arm.

In this project we were able to control the Robotic arm not only using the wired controls but with the help of Internet of Things which is the growing technology in recent times we successfully controlled the robotic arm using the IOT interface. This can be useful to various industrial applications where machines need to be controlled from distant places. This project not only responds to the controls sent but also records the movements and can perform the same tasks repeatedly reducing human efforts.

The Robotic arm consists of four servo motors and three dc gear motors. Four servo motors and a dc gear motor are used for the movement of the robotic arm. While the other two dc motors are used to the control of the wheels of the robotic arm. The picture of the robotic arm which is the final product of the project is given in the figure 3 . 


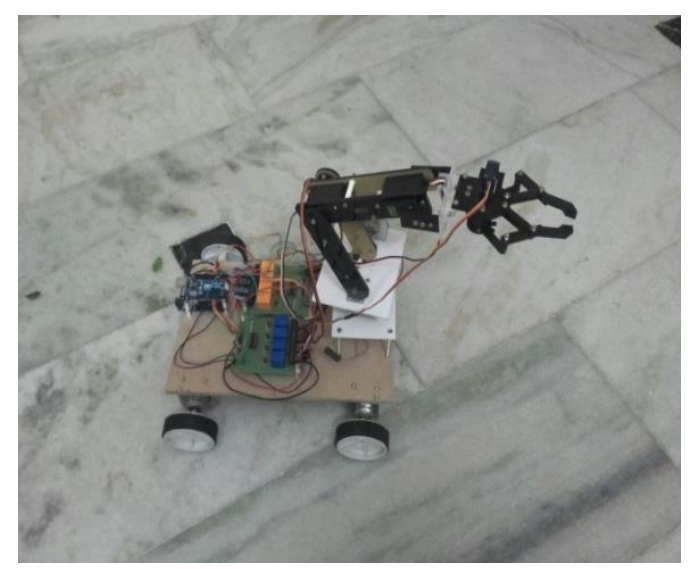

Fig. 5: Experimental setup of Wheeled robotic arm using IoT

The robotic arm can be controlled by the IoT using the mobile phone from anywhere in the world. Even if we lost our mobile we can control our robotic arm with the help of another mobile phone, what we needed is the installation of the blynk application. Also there will be no privacy lose due to the usage of the login id and password we created on the cloud. We can control our robotic arm with any mobile phones which have blynk application with our login details.

\section{Future Work}

1. This can be used for the space explorations.

2. In the medical field, we can do minor surgeries from far distance through IoT.

3. Detection and diffusion of Suspicious objects like mine bombs.

\section{Conclusion}

Brief summary of the most important conclusions from this study as follows:

(1) Lifting and moving heavy objects.

(2) Easy to move everywhere

(3) Increasing productivity, safety, efficiency, and quality of products.

(4) Achieving more accuracy than human beings.

(5) Easy to monitor and control things

(6) High security with password.
(7) We can control this, where ever we situated

\section{References}

[1] G.Ramakrishnaprabu; E.Elangovan;"'IoT Servomotor Enhanced Pick and Place Robo Arm" ISSN (Online): 2278 - 8875 Vol. 5, Issue 6, June 2016.

[2] Kaustubh Gawli ; Parinay Karande ; Pravin Belose; Tushar Bhadirke; Akansha Bhargava "Internet of things (iot) based robotic arm" e-ISSN: 2395-0056 Volume: 04 Issue: 03, Mar 2017.

[3] Carro.G.; Castro.M.; Sancristobal.E.; Diaz.G.; Mur.F; Latorre.M.; haparro.M.; Lopez-Rey.A; Salzmann.C.; Gillet.D, "The color of the light: A remote laboratory that uses a smart device that connects teachers and students" Global Engineering Education Conference (EDUCON), 2014 IEEE, vol., no., pp.854,860, 3-5 April 2014.

[4] Fernandez.G.C.; Gil.M.C.; Perez.F.M., "Remote robotic laboratory as nexus between students and real engineering," Interactive Collaborative Learning (ICL), 2012 15th International Conference on, vol., no.,pp.1,4, 26-28 Sept. 2012

[5] Carro Fernandez.G.; Gutierrez.S.M.; Ruiz.E.S.; Gil.M.C.; Perez.F.M., "Formation in robotics, the key to integration in industrial environments", Interactive Collaborative Learning (ICL), 2012 15th International Conference on, vol., no., pp.1,4, 26-28 Sept. 2012

[6] Song.G; Ding.F; Song.A, "A wireless power outlet system for smart homes", IEEE Transactions on Consumer Electronics, Vol. 54, No. 4, 2008.

[7] Kim.T.T; Poor.H.V., "Scheduling power consumption with price uncertainty", IEEE Transactions on Smart Grid, Vol. 2, No. 3, pp. 519-527, 2011

[8] S.V. Manikanthan , T. Padmapriya "An enhanced distributed evolved node-b architecture in 5G tele-communications network" International Journal of Engineering \& Technology (UAE), Vol 7 Issues No (2.8) (2018) 248-254.March2018.

[9] S.V. Manikanthan , T. Padmapriya "An enhanced distributed evolved node-b architecture in 5G tele-communications network" International Journal of Engineering \& Technology (UAE), Vol 7 Issues No (2.8) (2018) 248-254.March2018

[10] M. Rajesh, Manikanthan, "GET-UP-AND-GO EFFICIENTMEMETIC ALGORITHM BASED AMALGAM ROUTING PROTOCOL", International Journal of Pure and Applied Mathematics, ISSN NO:1314-3395, Vol-116, No. 21, Oct 2017.

[11] T. Padmapriya, V.Saminadan, "Performance Improvement in long term Evolution-advanced network using multiple input multiple output technique", Journal of Advanced Research in Dynamical and Control Systems, Vol. 9, Sp-6, pp: 990-1010, 2017. 